\title{
URANS and LES methodology for two-dimensional natural convection in a differentially heated cavity by BEM
}

\author{
L. Škerget \& J. Ravnik \\ University of Maribor, Faculty of Mechanical Engineering, Slovenia
}

\begin{abstract}
The paper considers the fundamental aspects of turbulence modelling for incompressible Boussinesq fluid flow and corresponding numerical models based on the boundary-domain integral equations. Two different solution methodologies of turbulent flow circumstances are considered, e.g. the classical statistical methodology known as Unsteady Reynolds-Averaged Navier-Stokes (URANS) equations and deterministic Large-Eddy-Simulation (LES) formulation. The velocity-vorticity formulation of the mean/filtered equations is applied, while the averaged/filtered pressure field is determined by solving the Poisson velocity equation. Chaotic natural convection in a differentially heated cavity of aspect ratio 4 with adiabatic horizontal walls is studied by both mentioned methodologies of the unsteady two-dimensional governing equations.

Keywords: turbulence, large eddy simulation, unsteady RANS, boundary element method.
\end{abstract}

\section{Introduction}

The set of partial differential equations governing the motion of viscous fluid is known as nonlinear Navier-Stokes equations. This equation system is generally considered to be the fundamental description for all laminar as well as turbulent flows, although some statistical averaging or deterministic filtering procedure is required in practice to predict the turbulence and simulate numerically the flow at higher Reynolds or Rayleigh number values due to the enormous computational effort needed. In the LES methodology the classical Smagorinsky model with Van Driest damping closed to cavity walls is considered, while in the URANS 
methodology the low Reynolds two equation $k-\epsilon$ Launder and Sharma turbulent model is applied.

The present investigation is concerned with URANS and LES of complex buoyancy-driven Boussinesq fluid flow in two dimensions in a 1:4 square differentially heated enclosure. For aspect ratio larger than or equal to 4 , the onset of unsteadiness is due to the instability of vertical boundary layers. Although the turbulent flow in the rectangular cavity is basically three-dimensional, twodimensional model can be applied in this case due to minor differences between the $3 \mathrm{D}$ and $2 \mathrm{D}$ flow field results.

\section{Governing mean/filtered flow equations}

\subsection{Primitive variables formulation}

The governing equations for the mean/filtered flow can be written in terms of effective momentum diffusivity $\nu_{e f}$ and thermal diffusivity $a_{e f}$, respectively, as follows

$$
\begin{gathered}
\frac{\partial \overline{v_{j}}}{\partial x_{j}}=0 \\
\rho_{o} \frac{D \overline{v_{i}}}{D t}=-\frac{\partial \bar{p}^{\star}}{\partial x_{i}}+\frac{\partial}{\partial x_{j}}\left(\rho_{o} 2 \nu_{e f} \overline{\dot{\varepsilon}_{i j}}\right)+\bar{\rho} g_{i}, \\
c_{o} \frac{D \bar{T}}{D t}=\frac{\partial}{\partial x_{j}}\left(c_{o} a_{e f} \frac{\partial \bar{T}}{\partial x_{j}}\right)+\overline{S_{T}},
\end{gathered}
$$

where the effective transport coefficient for the mean/filtered flow equations are given by the definitions, e.g. $\nu_{e f}=\nu+\nu_{t}, a_{e f}=a+a_{t}$, or similarly $\nu_{e f}=\nu+\nu_{s}$ and $a_{e f}=a+a_{s}$, respectively. The modified mean pressure term $\bar{p}^{\star}$ represents the sum of the static pressure and complementary volumetric part of Reynolds stress, such as

$$
\bar{p}^{\star}=\bar{p}+\frac{2}{3} \rho_{o} k
$$

while in the modified filtered pressure term $\bar{p}^{\star}$ the complementary spherical tensor part or the trace of subgrid-scale stress tensor has been lumped into the pressure by defining

$$
\bar{p}^{\star}=\bar{p}+\frac{1}{3} \rho_{o} \tau_{k k}^{R} .
$$

The momentum equation Eqn. (2) can be written in a form suitable for velocityvorticity formulation, e.g. in a vector form

$$
\begin{aligned}
\rho_{o} \frac{D \overline{\vec{v}}}{D t}= & -\operatorname{rot}\left(\eta_{e f} \overline{\vec{\omega}}\right)+2 \operatorname{grad} \overline{\vec{v}} \cdot \operatorname{grad} \eta_{e f}+2 \operatorname{grad} \eta_{e f} \times \overline{\vec{\omega}} \\
& -\operatorname{grad} \bar{p}^{\star}+\bar{\rho} \vec{g},
\end{aligned}
$$


The effective dynamic viscosity $\eta_{e f}$ and effective heat conductivity $k_{e f}$ can be given as a sum of a constant and variable part

$$
\eta_{e f}=\eta_{e f o}+\widetilde{\eta_{e f}}, \quad k_{e f}=k_{e f o}+\widetilde{k_{e f}},
$$

therefore the momentum and energy Eqns. (6) and (3) can be written in analogy to the basic conservation equations formulated for the constant material properties

$$
\begin{gathered}
\rho_{o} \frac{D \overline{\vec{v}}}{D t}=-\eta_{e f o} \operatorname{rot} \overline{\vec{\omega}}-\operatorname{grad} \bar{p}^{\star}+\bar{\rho} \vec{g}+\overline{\vec{f} m}, \\
c_{o} \frac{D \bar{T}}{D t}=k_{e f o} \Delta \bar{T}+\overline{S_{T}}+\overline{S_{T}^{m}},
\end{gathered}
$$

where the pseudo body force term $\overline{f^{m}}$ and pseudo heat source term $\overline{S_{T}^{m}}$, are introduced into the momentum Eqn. (8) and energy Eqn. (9), respectively, capturing the variable transport property effects, and given by expressions

$$
\overline{f_{i}^{m}}=-e_{i j k} \frac{\partial \overline{\omega_{k}}}{\partial x_{j}} \widetilde{\eta_{e f}}+e_{i j k} \frac{\partial \eta_{e f}}{\partial x_{j}} \overline{\omega_{k}}+2 \frac{\partial \eta_{e f}}{\partial x_{j}} \frac{\partial \overline{v_{i}}}{\partial x_{j}},
$$

while the pseudo heat source term is given by expression

$$
\overline{S_{T}^{m}}=\frac{\partial}{\partial x_{j}}\left(\widetilde{k_{e f}} \frac{\partial \bar{T}}{\partial x_{j}}\right) .
$$

Once the form of the eddy diffusivity coefficients are specified then the mean/filtered governing transport equations can be solved in the same manner as a laminar flow since the equations are the same except for augmented diffusivity coefficients. Though the turbulent flow problem has been reduced to a familiar system of partial differential transport equations, there remains the nontrivial task of determining how the eddy diffusivity coefficients vary with the flow field.

\subsection{Velocity-vorticity mean/filtered flow formulation}

With the mean/filtered vorticity vector $\bar{\omega}_{i}$ representing the curl of the velocity field $\overline{v_{i}}$

$$
\overline{\omega_{i}}=e_{i j k} \frac{\partial \overline{v_{k}}}{\partial x_{j}} \quad \text { and } \quad \frac{\partial \overline{\omega_{j}}}{\partial x_{j}}=0,
$$

the fluid motion computation scheme is partitioned into its kinematics, given by the elliptic mean/filtered velocity vector equation

$$
\frac{\partial^{2} \overline{v_{i}}}{\partial x_{j} \partial x_{j}}+e_{i j k} \frac{\partial \overline{\omega_{k}}}{\partial x_{j}}=0
$$

and kinetics given by mean/filtered vorticity transport equation, obtained as a curl of the mean/filtered momentum Eqn. (8), e.g., written in Cartesian tensor notation 
formulation

$$
\begin{array}{r}
\frac{\partial \overline{\omega_{i}}}{\partial t}+\frac{\partial \overline{v_{j}} \overline{\omega_{i}}}{\partial x_{j}}=\nu_{e f o} \frac{\partial^{2} \overline{\omega_{i}}}{\partial x_{j} \partial x_{j}}+\frac{\partial \overline{\omega_{j}} \overline{v_{i}}}{\partial x_{j}} \\
+\frac{1}{\rho_{o}} e_{i j k} \frac{\partial \bar{\rho} g_{k}}{\partial x_{j}}+\frac{1}{\rho_{o}} e_{i j k} \frac{\partial \overline{f_{k}^{m}}}{\partial x_{j}},
\end{array}
$$

which reduces for two-dimensional plane flow case, to the following scalar mean/filtered vorticity statement

$$
\frac{\partial \bar{\omega}}{\partial t}+\frac{\partial \overline{v_{j}} \bar{\omega}}{\partial x_{j}}=\nu_{e f o} \frac{\partial^{2} \bar{\omega}}{\partial x_{j} \partial x_{j}}-\frac{1}{\rho_{o}} e_{i j} \frac{\partial \bar{\rho} g_{i}}{\partial x_{j}}-\frac{1}{\rho_{o}} e_{i j} \frac{\partial \overline{f_{i}^{m}}}{\partial x_{j}} .
$$

The vorticity transport Eqn. (14) is highly nonlinear partial differential equation. Due to the buoyancy force and variable effective transport properties, acting as additional nonlinear vorticity source terms, the vorticity transport equation is coupled to the energy/mass and transport equations for the turbulence quantities, making the numerical computation procedure very challenging.

\section{Eddy-viscosity turbulence models}

\subsection{Two-equation LRN $k-\epsilon$ turbulence models}

In the $k-\epsilon$ turbulence models, the turbulent motion is characterized by two quantities, namely the turbulent kinetic energy $k$ and the turbulent energy dissipation rate $\epsilon$, e.g. given by relations

$$
k=\frac{1}{2} \overline{v_{i}^{\prime} v_{i}^{\prime}}, \quad \epsilon=\nu \overline{\frac{\partial v_{i}^{\prime}}{\partial x_{j}} \frac{\partial v_{i}^{\prime}}{\partial x_{j}}}
$$

while the turbulent viscosity is given by the Kolmogorov-Prandtl relation

$$
\eta_{t}=C_{\eta} \rho_{o} f_{\eta} \frac{k^{2}}{\epsilon}
$$

which relates the eddy viscosity directly to the turbulence variables, $k$ and $\epsilon$, and where $C_{\eta}=0.09$ is an empirical constant. The values of $k$ and $\epsilon$ come directly from the differential transport equations for the turbulent kinetic energy and the eddy dissipation rate

$$
\begin{gathered}
\rho_{o} \frac{D k}{D t}=\frac{\partial}{\partial x_{j}}\left[\left(\eta_{o}+\frac{\eta_{t}}{\sigma_{k}}\right) \frac{\partial k}{\partial x_{j}}\right]+P_{k}-\rho_{o} \widetilde{\epsilon}-\rho_{o} D, \\
\rho_{o} \frac{D \widetilde{\epsilon}}{D t}=\frac{\partial}{\partial x_{j}}\left[\left(\eta_{o}+\frac{\eta_{t}}{\sigma_{\epsilon}}\right) \frac{\partial \widetilde{\epsilon}}{\partial x_{j}}\right]+C_{\epsilon 1} f_{\epsilon 1} \frac{\widetilde{\epsilon}}{k} P_{k}-C_{\epsilon 2} f_{\epsilon 2} \rho_{o} \frac{\widetilde{\epsilon}^{2}}{k}+\rho_{o} E,
\end{gathered}
$$


where the model variable $\tilde{\epsilon}$ is defined as

$$
\epsilon \equiv \widetilde{\epsilon}+D \quad \text { with } \quad D=2.0 \nu_{o}\left(\frac{\partial k^{1 / 2}}{\partial x_{j}}\right)^{2} .
$$

Since the dissipation rate at the wall is equal to

$$
\left.\left.\epsilon\right|_{\text {wall }} \equiv D\right|_{\text {wall }}=\left.2.0 \nu_{o}\left(\frac{\partial k^{1 / 2}}{\partial x_{j}}\right)^{2}\right|_{\text {wall }},
$$

the variable $\tilde{\epsilon}$ is zero at the wall which simplifies the specification of wall boundary conditions, i.e.

$$
k=\widetilde{\epsilon}=0 .
$$

Further, such definition of new variable $\widetilde{\epsilon}$ also gives rise to an extra term $E$ in the $\widetilde{\epsilon}$ transport equation

$$
E=2.0 \nu_{o} \nu_{t}\left(\frac{\partial^{2} \overline{v_{i}}}{\partial x_{j} \partial x_{k}}\right)^{2}
$$

The turbulent kinetic energy production term $P_{k}$ is due to viscous and buoyancy forces and is modelled, e.g. by the following relation

$$
P_{k}=2 \eta_{t} \overline{\dot{\varepsilon}_{i j}} \frac{\partial \overline{v_{i}}}{\partial x_{j}}-\eta_{t} g_{i} \frac{\partial \rho / \partial x_{i}}{\sigma_{\rho} \rho} .
$$

The damping functions are expressed as functions of the local turbulence Reynolds number $R e_{t}$ as follows:

$$
\begin{gathered}
f_{\eta}=\exp \left[-\frac{3.4}{\left(1+0.02 R e_{t}\right)^{2}}\right] \quad \text { and } \quad R e_{t}=\frac{\rho k^{2}}{\eta \epsilon}, \\
f_{\epsilon 1}=1 \quad \text { and } \quad f_{\epsilon 2}=1.00-0.3 \exp \left(-R e_{t}^{2}\right),
\end{gathered}
$$

with $C_{\epsilon 1}=1.44, C_{\epsilon 2}=1.92, \sigma_{k}=1.0$, and $\sigma_{\epsilon}=1.3$, while $\sigma_{\rho}=0.9$, respectively.

\subsection{Subgrid-scale closure/modeling}

One of the most popular Boussinesq eddy-viscosity subgrid closure model is due to Smagorinsky, e.g. which correlates $\tau_{i j}^{R}$ to the large-scale strain-rate tensor $\overline{\dot{\varepsilon}_{i j}}$

$$
\tau_{i j}^{R}=-2 \eta_{s} \overline{\dot{\varepsilon}_{i j}}+\frac{1}{3} \rho_{o} \tau_{k k}^{R} \delta_{i j} .
$$

The subgrid viscosity $\eta_{s}$ can be expressed as

$$
\nu_{s} \propto l_{s} v_{s}
$$


where $l_{s}$ is the length scale of the unresolved flow and $v_{s}$ its velocity scale. The subgrid viscosity can be expressed as

$$
l_{s}=\triangle=(\triangle \Omega)^{1 / 3},
$$

where $\triangle \Omega$ is the volume of the computational internal cell. The velocity scale is related to the gradients of $\overline{v_{i}}$ and it is defined as

$$
v_{s}=l_{s} \overline{\dot{\gamma}}
$$

where $\overline{\dot{\gamma}}$ is the deformation velocity of the resolved flow or the magnitude of the large-scale strain-rate tensor $\overline{\dot{\varepsilon}_{i j}}$ defined as

$$
\overline{\dot{\gamma}}=\left(2 \overline{\dot{\varepsilon}_{i j}} \overline{\dot{\varepsilon}_{i j}}\right)^{1 / 2} \quad \text { and } \quad \overline{\dot{\varepsilon}_{i j}}=\frac{1}{2}\left(\frac{\partial \overline{v_{i}}}{\partial x_{j}}+\frac{\partial \overline{v_{j}}}{\partial x_{i}}\right) .
$$

Finally the subgrid-scale viscosity is defined as

$$
\nu_{s}=\left(C_{s} \triangle\right)^{2} \overline{\dot{\gamma}},
$$

where $C_{s}$ is the Smagorinsky constant. It is found that values of $C_{s}$ vary from 0.065 to 0.2 for isotropic turbulence. Furthermore, $C_{s}$ has to be reduced in the near wall region to account for the turbulence anisotropy. Thus, the correct distribution of $C_{s}$ in the near wall region is obtained by using so-called damping functions, e.g. the most often used is the van Driest damping function

$$
C_{s}=C_{s o}\left[1-\exp \left(-R e_{\tau} / 25\right)\right]^{2} .
$$

The subgrid-scale heat flux $\overline{v_{j} T}$ can be modelled as simple gradient diffusion hypothesis

$$
\overline{v_{j} T}=-\frac{\nu_{s}}{P r_{t}} \frac{\partial \bar{T}}{\partial x_{j}}
$$

\section{Boundary-domain integral equations}

The kinematics of plane motion is given by two scalar equations as follows:

$$
c(\xi) \overline{v_{i}}(\xi)+\int_{\Gamma} \overline{v_{i}} q d \Gamma=\int_{\Gamma} \frac{\partial v_{i}}{\partial n} u^{\star} d \Gamma+e_{i j} \int_{\Gamma} \bar{\omega} n_{j} u^{\star} d \Gamma-e_{i j} \int_{\Omega} \bar{\omega} q_{j}^{\star} d \Omega .
$$

where $u^{\star}$ stands for the elliptic Laplace fundamental solution and $q^{\star}$ is its normal derivative, e.g. $q^{\star}=\partial u^{\star} / \partial n=\vec{q}^{\star} \cdot \vec{n}$, while the vector flux variable is defined as $q_{i}^{\star}=\partial u^{\star} / \partial x_{i}$. The most important issues in numerical modelling of incompressible fluid flow is to obtain a divergence free final solution for the velocity and vorticity vector field functions. Thus, the proper kinematic integral representation should preserve the compatibility and restriction conditions for the velocity and vorticity field functions. Accounting for the additional compatibility 
and restriction conditions for velocity and vorticity fields, e.g. $\vec{\omega}=\operatorname{rot} \vec{v}$ and $\operatorname{div} \vec{v}=0$, the following boundary integral representation for the general flow situation can be stated for the two-dimensional plane flow kinematic case as follows

$$
c(\xi) \overline{v_{i}}(\xi)+\int_{\Gamma} \overline{v_{i}} q^{\star} d \Gamma=e_{i j} \int_{\Gamma} \overline{v_{j}} q_{t}^{\star} d \Gamma-e_{i j} \int_{\Omega} \bar{\omega} q_{j}^{\star} d \Omega .
$$

Using unique feature of global integral representation for boundary vorticity values, the vector eq.(36) has to be written in its tangential form in order to obtain an appropriate non-singular implicit system of equations for unknown boundary vorticity values.

Considering the kinetics in an integral representation one has to take into account the parabolic diffusion-convection character of the vorticity transport equation. With the use of the linear parabolic diffusion operator the vorticity equation can be formulated as a scalar inhomogeneous parabolic diffusion equation as follows:

$$
\begin{gathered}
c(\xi) \bar{\omega}\left(\xi, t_{F}\right)+\int_{\Gamma} \bar{\omega} Q^{\star} d \Gamma=\frac{1}{\eta_{o}} \int_{\Gamma}\left(\eta_{o} \frac{\partial \bar{\omega}}{\partial n}-\rho_{o} v_{n} \bar{\omega}+\rho g_{t}\right) U^{\star} d \Gamma \\
+\frac{1}{\eta_{o}} \int_{\Omega}\left(\rho_{o} \overline{v_{j} \omega}+e_{i j}\left(\rho g_{i}+\overline{f_{i}^{m}}\right)\right) Q_{j}^{\star} d \Omega+\int_{\Omega} \bar{\omega}_{F-1} u_{F-1}^{\star} d \Omega,
\end{gathered}
$$

where a constant variation of all field functions within the individual time increment $\Delta t=t_{F}-t_{F-1}$ is assumed, e.g. the values at $t=t_{F}$ are considered for each time step, where $v_{n}$ and $g_{t}$ are the normal velocity, and the tangential gravity, respectively, e.g. $v_{n}=\vec{v} \cdot \vec{n}, g_{t}=\vec{g} \cdot \vec{t}=-e_{i j} g_{i} n_{j}$.

The integral representation of the heat energy diffusion-convection transport equation is derived considering the linear parabolic diffusion differential operator and therefore the equation may be rewritten in the form

$$
\begin{gathered}
c(\xi) \bar{T}\left(\xi, t_{F}\right)+\int_{\Gamma} \bar{T} Q^{\star} d \Gamma=\frac{1}{k_{o}} \int_{\Gamma}\left(k_{o} \frac{\partial \bar{T}}{\partial n}-c_{o} \overline{v_{n}} \bar{T}\right) U^{\star} d \Gamma \\
+\frac{1}{k_{o}} \int_{\Omega}\left(c_{o} \overline{v_{j}} \bar{T}+\overline{S_{T}^{m}}\right) Q_{j}^{\star} d \Omega+\int_{\Omega} \bar{T}_{F-1} u_{F-1}^{\star} d \Omega,
\end{gathered}
$$

where a constant variation of all field functions within the individual time increment $\Delta t=t_{F}-t_{F-1}$ is assumed.

\section{Numerical aspects/iterative strategy}

The boundary element implementation of the two equation $k-\epsilon$ low-Reynoldsnumber model is basically straightforward since the transport equations for the 
turbulent quantities are of the familiar diffusion-convection type. The details of the iterative scheme can be formulated as follows. For the given velocity field $\vec{v}$ the $k$ and $\epsilon$ equations have to be solved. The first point of the iterative scheme is that the equations for $k$ and $\epsilon$ are coupled iteratively. Therefore, the nonlinear transport equation is solved for $k$ first assuming that $\epsilon$ is known and then the computed value of $k$ is used to solve the nonlinear transport equation for $\epsilon$. Since it is not desired to deal with problems with a negative production term, the term $P_{k}$ is kept constant in Eqn. (19) and also production term is kept constant in Eqn. (18). In the innermost iterative loop the linearized $k$ equation is solved.

Next the linearized $\epsilon$ equation is solved. Now, the iterative scheme can be written in detail, as follows:

1. Compute $P_{k}$ and $P_{\epsilon}$

2. Repeat until convergence for $\nu_{t}$

2.1 Solve for $k$ :

2.1.1 Solve linearized $k$ equation

$2.1 .2 k_{i}=u r \cdot k_{i}+(1-u r) \cdot k_{i-1}$

2.2 Check convergence for $k$. If not, go to 2.1.

2.3 Update $\nu_{t}$ using Eqn. (17)

2.4 Solve for $\epsilon$ :

2.4.1 Solve linearized $\epsilon$ equation

$2.4 .2 \epsilon_{i}=u r \cdot \epsilon_{i}+(1-u r) \cdot \epsilon_{i-1}$

2.4 Check convergence for $\epsilon$. If not, go to 2.4.

2.5 Update $\nu_{t}$ using Eqn. (17)

3. Check convergence for $\nu_{t}$. If not, go to 2 .

The main advantages of the Smagorinsky model are its simplicity and its stability. Whether filtering is introduced or not, the LES equations with subgridscale eddy viscosity model are solved numerically for the time evolution of the LES field functions. This involves discretization in space and time, which introduces differences between the differential equations and their numerical equivalent. The solution iterative strategy is to solve for large scale velocity $\vec{v}$ and vorticity $\vec{\omega}$ field functions and then to compute subgrid-scale eddy viscosity until convergence, repeating the iterative process if needed. The solution scheme is as follows:

1. Solve the filtered Navier-Stokes LES equations

1.1 Update subgrid-scale eddy viscosity $\nu_{s}$

2. Check convergence for $\vec{\omega}$. If not, go to 1 .

\section{Two-dimensional natural convection in a differently heated cavity of aspect ratio 4}

We consider a cavity of height $H=4$ and width $W=1$, aspect ratio $A=H / W=4$, filled with a Newtonian viscous fluid. It is submitted to a temperature difference $\triangle T=T_{h}-T_{c}>0$ at the vertical walls, with uniform temperatures $T_{h}=0.5$ and $T_{c}=-0.5$, respectively, while the top and bottom 

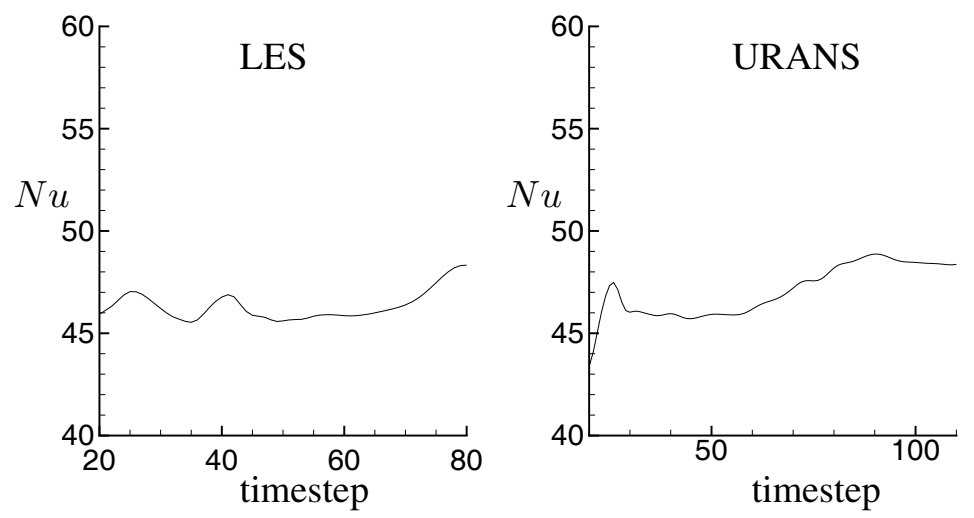

Figure 1: Development of heat flux expressed by Nusselt number.

walls are adiabatic.Two non-uniform numerical models are considered consisting of $M=40 \times 160$ and $M=60 \times 240$ macro elements with the aspect ratio of $1: 4$ in the $x$ - and $y$-direction. Three-node quadratic boundary elements and nine-node quadratic internal cells are applied. The time dependent numerical simulation is performed with the dimensionless time step $\triangle t=5 \cdot 10^{-4}$.

Turbulent natural convection in a differently heated air-filled cavity of aspect ratio 4 with adiabatic horizontal walls is investigated by large eddy simulation LES and unsteady mean flow URANS numerical integration of the unsteady two-dimensional governing equations. In order to approach chaotic flows which exhibits randomness in space as well as in time, simulations for different Rayleigh number values, e.g. $R a=6.4 \cdot 10^{8}$, and for Prandtl number value $\operatorname{Pr}=0.71$ are performed.
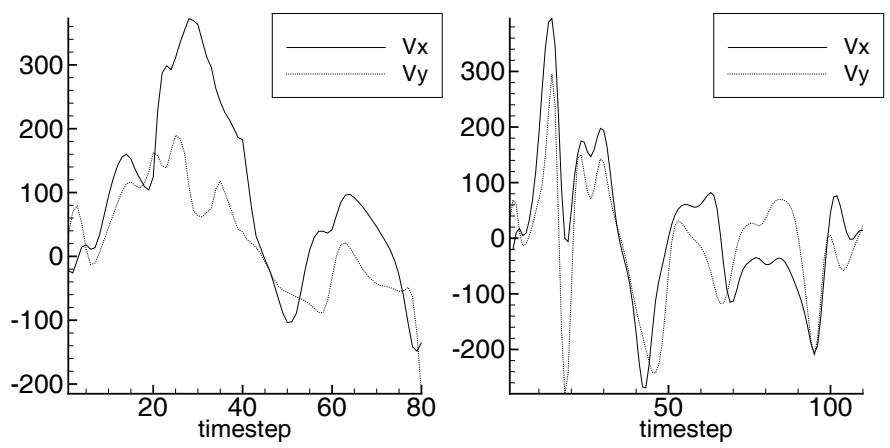

Figure 2: Time traces of horizontal and vertical velocity components recorded at location $(0.75,0.25)$. LES turbulence model (left), URANS turbulence model (right). $R a=6.4 \cdot 10^{8}$ 

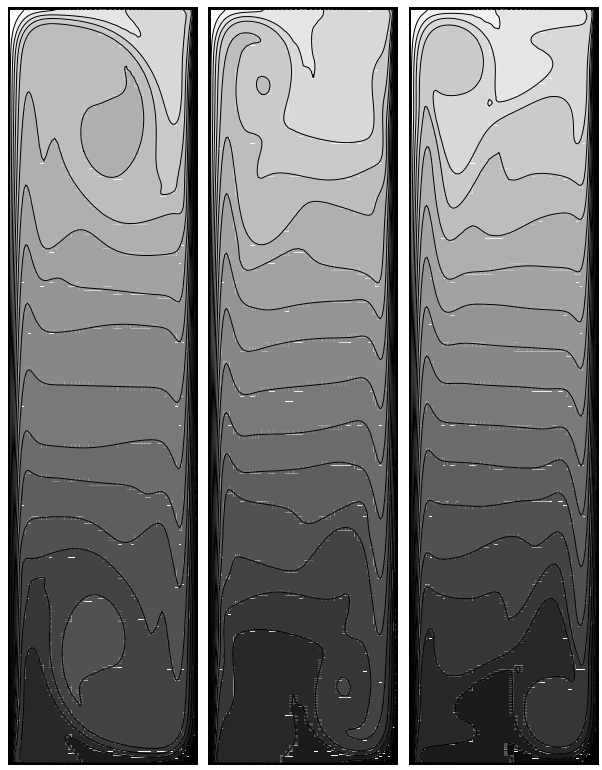

Figure 3: The temperature field at $R a=6.4 \cdot 10^{8}$. Three instantaneous timesteps are shown. Turbulence is modelled by URANS approach.

Figure 1 shows heat flux expressed by Nusselt number. The value of 48.3 was recorded at the final time step. This compares well with $N u=49.2$, which was reported by Xin and Le Quéré [9] and with $N u=49.08$ reported by Ravnik et al. [8]. Figure 2 shows the time traces of horizontal and vertical velocity components recorded at location $(0.75,0.25)$ for both turbulence models. The location is inside the vortex, which is located in the corner of the enclosure. We observe rapid changes in the velocity field. Finally, Figures 3 and 4 show instantaneous temperature contours for several time instants. One can readily observe the unsteady nature of the flow.

\section{Conclusion}

In this work a numerical procedure based on the boundary element method for the simulation of turbulent buoyancy-driven two-dimensional fluid flow in a differentially heated air-filled cavity of aspect ratio 4 is investigated. The flow circumstances for the Rayleigh number value $R a=6.4 \cdot 10^{8}$ are presented in this paper. Relatively course mesh consisting of $40 \times 160$ macro elements is used in the numerical model. The periodic oscillations of the field functions in the downstream parts of the boundary layers in the form of Tollmien-Schlichting waves are detected. Rather large fluctuations are observed in the cavity corners where the flow is very chaotic. The main cavity core is still well stratified and basically motionless, therefore the flow is still far from being turbulent. With increasing $R a$ the cavity core becomes deorganised and chaotic. Finer mesh, e.g. 

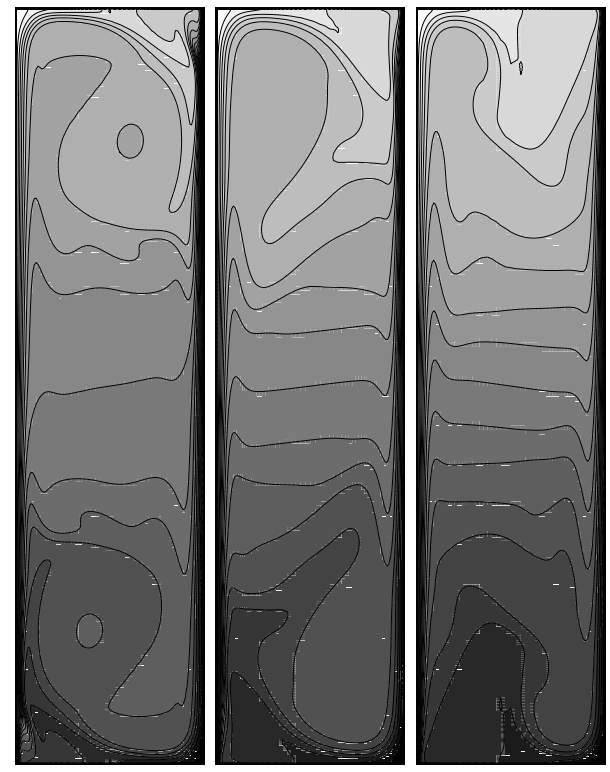

Figure 4: The temperature field at $R a=6.4 \cdot 10^{8}$. Three instantaneous timesteps are shown. Turbulence is modelled by LES approach.

consisting of $60 \times 240$ macro elements, have to be used to accurately simulate turbulent flow structures at higher Ra number values.

Low Reynolds number $k-\epsilon$ and LES turbulence modelling methodologies have been considered. Iterative strategy of highly nonlinear and coupled governing equations is discussed. The main goal of the paper is to increase the applicability of BEM technique to solve real turbulent fluid flow problems.

\section{References}

[1] G. Biswas and V. Eswaran (2002) Turbulent Flows: Fundamentals, Experiments and Modeling. Alpha Science International Ltd., Pangbourne, UK.

[2] Tennekes, H. and Lumley, J.L.: A First Course in Turbulence. MIT Press, (1972).

[3] Abe, K., Kondoh, T. and Nagano, Y.: A New Turbulence Model for Predicting Fluid Flow and Heat Transfer in Separating and Reattaching Flows - I. Flow Field Calculations. Int. Journal of Heat and Mass Transfer, Vol. 37, pp. 139151, (1994).

[4] L. Škerget and J. Ravnik (2009) BEM simulation of compressible fluid flow in an enclosure induced by thermoacoustic waves. Eng. Anal. Boundary Elements, 33, 561-571. 
[5] L. Škerget and J. Ravnik (2010) Simulation of fluid flow by BEM, in Recent Developments in Boundary Element Methods : a Volume to Honour Professor John T. Katsikadelis, WIT Press, Southampton, pp. 213-226.

[6] L. Škerget and J. Ravnik (2010) Solution of velocity-vorticity URANS by BEM, in Boundary elements and other mesh reduction methods XXXII, (WIT transactions on modelling and simulation), WIT Press, Southampton, pp. 29-40.

[7] L. Škerget and J. Ravnik (2009) Boundary element analysis of general laminar and turbulent fluid flow problems, in 2nd South-East European Conference on Computational Mechanics. Institute of Structural Analysis \& Seismic Research, National Technical University of Athens.

[8] J. Ravnik, L. Škerget and M. Hriberšek (2006) 2D velocity vorticity based LES for the solution of natural convection in a differentially heated enclosure by wavelet transform based BEM and FEM, Eng. Anal. Boundary Elements, 30, 671-686.

[9] S. Xin and P. Le Quéré (1995) Direct numerical simulations of twodimensional chaotic natural convection in a differentially heated cavity of aspect ratio 4, J. Fluid Mech., 304, 87-118. 